\title{
Demonstration of near infrared gas sensing using gold nanodisks on functionalized silicon
}

\author{
P.J. Rodríguez-Cantó, ${ }^{1, *}$ M. Martínez-Marco, ${ }^{1}$ F. J. Rodríguez-Fortuño, ${ }^{1}$ B. Tomás- \\ Navarro, ${ }^{1}$ R. Ortuño, ${ }^{1}$ S. Peransí-Llopis, ${ }^{2}$ A. Martínez ${ }^{1}$ \\ 1: Nanophotonics Technology Center, Universidad Politécnica de Valencia, Camino de Vera s/n, 46022, Valencia, \\ Spain \\ ${ }^{2}$ : Instituto de Reconocimiento Molecular, Dpto de Química, Universidad Polítecnica de Valencia, Camino de Vera \\ $s / n, 46022$ Valencia, Spain \\ pjrodrig@ntc.upv.es
}

\begin{abstract}
In this work, we demonstrate experimentally the use of an array of gold nanodisks on functionalized silicon for chemosensing purposes. The metallic nanostructures are designed to display a very strong plasmonic resonance in the infrared regime, which results in highly sensitive sensing. Unlike usual experiments which are based on the functionalization of the metal surface, we functionalized here the silicon substrate. This silicon surface was modified chemically by buildup of an organosilane selfassembled monolayer (SAM) containing isocyanate as functional group. These groups allow for an easy surface regeneration by simple heating, thanks to the thermally reversible interaction isocyanate-analyte, which allows the cyclic use of the sensor. The technique showed a high sensitivity to surface binding events in gas and allowed the surface regeneration by heating of the sensor at $150^{\circ} \mathrm{C}$. A relative wavelength shift $\Delta \lambda_{\max } / \lambda_{0}=0.027$ was obtained when the saturation level was reached.
\end{abstract}

(C2011 Optical Society of America

OCIS codes: (250.5403) Plasmonics; (240.6680) Surface plasmons; (130.6010) Sensors; (220.4241) Nanostructure fabrication; (300.6340) Spectroscopy, infrared.

\section{References and links}

1. W. L. Barnes, A. Dereux, and T. W. Ebbesen, "Surface plasmon subwavelength optics,” Nature 424(6950), 824830 (2003).

2. S. A. Maier, M. L. Brongersma, P. G. Kik, S. Meltzer, A. A. G. Requicha, and H. A. Atwater, "Plasmonics-A Route to Nanoscale Optical Devices,” Adv. Mater. 13(19), 1501-1505 (2001).

3. S. Link, and M. A. El-Sayed, "Optical properties and ultrafast dynamics of metallic nanocrystals," Annu. Rev. Phys. Chem. 54(1), 331-366 (2003).

4. K. A. Willets, and R. P. Van Duyne, "Localized surface plasmon resonance spectroscopy and sensing,” Annu. Rev. Phys. Chem. 58(1), 267-297 (2007).

5. J. N. Anker, W. P. Hall, O. Lyandres, N. C. Shah, J. Zhao, and R. P. Van Duyne, "Biosensing with plasmonic nanosensors," Nat. Mater. 7(6), 442-453 (2008).

6. J. Zhao, X. Zhang, C. R. Yonzon, A. J. Haes, and R. P. Van Duyne, "Localized surface plasmon resonance biosensors,” Nanomedicine (Lond) 1(2), 219-228 (2006).

7. D. R. Shankaran, K. V. Gobi, and N. Miura, "Recent advancements in surface plasmon resonance immunosensors for detection of small molecules of biomedical, food and environmental interest,” Sens. Actuators B Chem. 121(1), 158-177 (2007).

8. N. Miura, K. Ogata, G. Sakai, T. Uda, and N. Yamazoe, "Detection of morphine in ppb range by using SPR (Surface-plasmon resonance) immunosensor," Chem. Lett. 26(8), 713-714 (1997).

9. D. R. Shankaran, K. Matsumoto, K. Toko, and N. Miura, "Development and comparison of two immunoassays for the detection of 2,4,6-Trinitrotoluene (TNT) based on surface plasmon resonance," Sens. Actuators B Chem. 114(1), 71-79 (2006).

10. S. Cosnier, "Biomolecule immobilization on electrode surfaces by entrapment or attachment to electrochemically polymerized films. A review,” Biosens. Bioelectron. 14(5), 443-456 (1999).

11. J. W. Lee, S. J. Sim, S. M. Cho, and J. Lee, "Characterization of a self-assembled monolayer of thiol on a gold surface and the fabrication of a biosensor chip based on surface plasmon resonance for detecting anti-GAD antibody,” Biosens. Bioelectron. 20(7), 1422-1427 (2005). 
12. S. S. Mark, N. Sandhyarani, C. Zhu, C. Campagnolo, and C. A. Batt, "Dendrimer-functionalized self-assembled monolayers as a surface plasmon resonance sensor surface,” Langmuir 20(16), 6808-6817 (2004).

13. K. Kato, C. M. Dooling, K. Shinbo, T. H. Richardson, F. Kaneko, R. Tregonning, M. O. Vysotsky, and C. Hunter, "Surface plasmon resonance properties and gas response in porphyrin Langmuir-Blodgett films," Eng. Aspects 198-200, 811-816 (2002).

14. W. Senaratne, L. Andruzzi, and C. K. Ober, "Self-assembled monolayers and polymer brushes in biotechnology: current applications and future perspectives," Biomacromolecules 6(5), 2427-2448 (2005).

15. M. E. Stewart, C. R. Anderton, L. B. Thompson, J. Maria, S. K. Gray, J. A. Rogers, and R. G. Nuzzo, "Nanostructured plasmonic sensors," Chem. Rev. 108(2), 494-521 (2008).

16. L. Yin, Y. Liu, Z. Ke, and J. Yin, "Preparation of a blocked isocyanate compound and its grafting onto styrene-b(ethylene-co-1-buteno)-b-styrene triblock copolymer,” Eur. Polym. J. 45(1), 191-198 (2009).

17. K. Suyama, H. Iriyama, M. Shirai, and M. Tsunooka, "Curing systems using photolysis of carbamoyloxyimino groups and thermally regenerated isocyanate groups,” J. Photopolym. Sci. Technol. 14(2), 155-158 (2001).

18. S. Patskovsky, A. V. Kabashin, M. Meunier, and J. H. T. Luong, "Near-infrared surface plasmon resonance sensing on a silicon platform," Sens. Actuators B Chem. 97(2-3), 409-414 (2004).

19. D. J. Shelton, D. W. Peters, M. B. Sinclair, I. Brener, L. K. Warne, L. I. Basilio, K. R. Coffey, and G. D. Boreman, "Effect of thin silicon dioxide layers on resonant frequency in infrared metamaterials," Opt. Express 18(2), 1085-1090 (2010).

20. V. Bhalla, S. Carrara, C. Stagni, and B. Samorì, "Chip cleaning and regeneration for electrochemical sensor arrays,” Thin Solid Films 518(12), 3360-3366 (2010).

21. M. D. Malinsky, K. L. Kelly, G. C. Schatz, and R. P. Van Duyne, "Chain length dependence and sensing capabilities of the localized surface plasmon resonance of silver nanoparticles chemically modified with alkanethiol self-assembled monolayers,” J. Am. Chem. Soc. 123(7), 1471-1482 (2001).

22. M. J. S. Spencer, and G. L. Nyberg, “Adsorption of silane and methylsilane on gold surfaces,” Surf. Sci. 573(2), 151-168 (2004).

23. R. Gradess, R. Abargues, A. Habbou, J. Canet-Ferrer, E. Pedrueza, A. Russell, J. L. Valdés, and J. P. MartínezPastor, "Localized surface plasmon resonance sensor based on Ag-PVA nanocomposites thin films," J. Mater. Chem. 19(48), 9233-9240 (2009).

24. A. G. Brolo, R. Gordon, B. Leathem, and K. L. Kavanagh, "Surface plasmon sensor based on the enhanced light transmission through arrays of nanoholes in gold films,” Langmuir 20(12), 4813-4815 (2004).

25. E. Mauriz, A. Calle, A. Montoya, and L. M. Lechuga, "Determination of environmental organic pollutants with a portable optical immunosensor,” Talanta 69(2), 359-364 (2006).

26. Q. Yu, S. Chen, A. D. Taylor, J. Homola, B. Hock, and S. Jiang, "Detection of low-molecular-weight domoic acid using surface plasmon resonance sensor,” Sens. Actuators B Chem. 107(1), 193-201 (2005).

27. X. Cui, F. Yang, Y. Sha, and X. Yang, "Real-time immunoassay of ferritin using surface plasmon resonance biosensor," Talanta 60(1), 53-61 (2003).

\section{Introduction}

Surface plasmons (SPs) are electromagnetic waves that propagate at the interface between a metal and a dielectric medium. The electromagnetic field is trapped on the surface owing to its interaction with the free electrons in the metal and it decays exponentially with distance from the surface [1,2]. As a consequence, a strong field enhancement is expected just on the metal surface, which makes the SP very sensitive to variations of the optical properties of the dielectric material. In a flat surface the SP dispersion relation exclusively depends on the electric permittivity of the metallic and dielectric media. However, metallic nanoparticles in contact with dielectric media will support localized SP resonances (LSPR) whose resonant frequencies will depend also on the shape and size of the particle [3]. At those frequencies, the electromagnetic field will be strongly localized at the nanoparticle surface. This property has enabled a wide range of practical applications, being one of the most preferred sensing platforms for gas detection and biosensing. LSPR sensors are based thus on the measurement of variations in the LSPR frequencies due to small changes in the refractive index of the surrounding medium (substrate, solvent, and adsorbates) of the metal particle [4-6]. This kind of sensors has demonstrated good performance in terms of sensitivity, selectivity, reliability in analyses and low cost [7].

In order to use metallic nanoparticles for sensing purposes, a number of different approaches have been demonstrated for immobilization of different molecules on the metal surfaces, such as physical adsorption [8,9], embedding in polymers [10], self-assembly [11,12], or Langmuir-Blodgett deposition [13]. Among them, use of self-assembly monolayers (SAMs) for coating metal surfaces before immobilization is of considerable 
interest because they help to minimize the non-specific adsorption problem and to introduce reactive groups for specific immobilization [7]. These nanometer-thick layers are easily fabricated from commercially available substances. The most used methods are based on the formation of SAMs on the metallic (gold) surfaces containing free anchor groups such as thiols, amines, or acids for covalent binding of analytes [11,14]. The possibility for having different functional end groups provides a high degree of flexibility in terms of the types of analytes that can be attached. In addition to this, recent advances in nanofabrication methodologies have led to the realization of metal nanostructures based on nanoparticles, nanoholes, and other components with precisely controlled shapes, sizes and spacing [15]. Regeneration process of the functionalized metal surface is usually achieved by using chemical reactants in the $\mathrm{pH}$ range from highly acidic to basic. However, they can affect the binding ability of the functionalized surface and could even damage the nanostructure by erosion of the metal, resulting in a general diminished lifetime of the chip.

In this work, we experimentally demonstrate the use of gold nanodisks on functionalized silicon as a suitable platform for sensing of SAMs and subsequent binding of organic molecules. The metallic nanodisks are designed to support SPRs in the infrared regime, specifically at wavelengths around $1.5 \mu \mathrm{m}$. Functionalization methods reported in literature are based on the concept of molecular self-assembly of thiol- or disulfide molecules on the metal surface [11]. Instead of this common metal functionalization, here we chemically modify the silicon substrate with an organosilane containing isocyanate groups as active sites. These groups possess high reactivity with amine molecules at room temperature [16,17] and allow the regeneration of the isocyanate and the amine at elevated temperature. Evaluation of the sensing capability of this configuration is performed exposing the sensor to ethylenediamine vapour and characterizing the resulting shift of the LSPR. A key feature of this sensor is that, in contrast to other approaches in which the functionalization is carried out on the metal surface, it allows the repetitive use of the sensor by simple heating, avoiding this way the use of chemical reactants. In addition, the use of a silicon platform paves the way towards massive development of low-cost and portable miniaturized sensors [18].

\section{Design and fabrication of the gold nanodisks}

Metallic gold nanodisks on a silicon substrate, depicted schematically in Fig. 1(a), were designed to show a strong localized surface plasmon resonance (LSPR) in the infrared regime. The design was performed using numerical CST Microwave Studio time domain simulations to obtain the transmission spectral response of the sample. The thickness of the gold nanodisks was $20 \mathrm{~nm}$. In the fabrication process, a $3 \mathrm{~nm}$ thick chrome layer was deposited before the gold to increase the adherence of the nanodisks, and this was considered in the numerical design. Gold and chrome were modeled using Drude's model, with $\varepsilon_{\infty}=9$ (1) and $\omega_{p}=1.33 \times 10^{16} \mathrm{rad} / \mathrm{s}\left(6.69 \times 10^{15} \mathrm{rad} / \mathrm{s}\right)$ for gold (chrome), $\gamma=1 \times 10^{14} \mathrm{~s}^{-1}$ in both. Infrared metamaterials fabricated on silicon substrates exhibit a high degree of sensitivity to very thin layers of low permittivity materials between metallic elements and the underlying substrate. Therefore, native oxide layers on the silicon surface which are normally ignored in numerical simulations, were here taken into account by including a $2 \mathrm{~nm}$ layer with index 1.45 to produce more accurate predictions [19]. The resulting simulated transmission spectrum of the design is shown in Fig. 1(b), showing a LSPR at around $1.5 \mu \mathrm{m}$. The diameter of the nanodisks is $d=200 \mathrm{~nm}$ and the array periodicity is a $=400 \mathrm{~nm}$. In the spectral range under consideration, we did not observed significant coupling effects between adjacent nanodisks, so the periodicity does not play a role in the sensing performance.

The plasmonic nature of the nanodisk resonance makes the spectral response of the sample highly sensitive to minimal changes in the nanodisk surface, where the electric field at resonance is strong. The electric field [see Fig. 1(c)] is also strong at the surface of the silicon substrate near the nanoparticles, which opens the possibility of sensing monolayers deposited in the substrate rather than in the nanoparticle. The numerical simulated sensitivity to 
variations in the surrounding refractive index is $84 \mathrm{~nm} / \mathrm{RIU}$ and the Figure-of-Merit FOM = sensitivity (nm/RIU) / full-width-at-half-maximum (nm) is 0.19 .
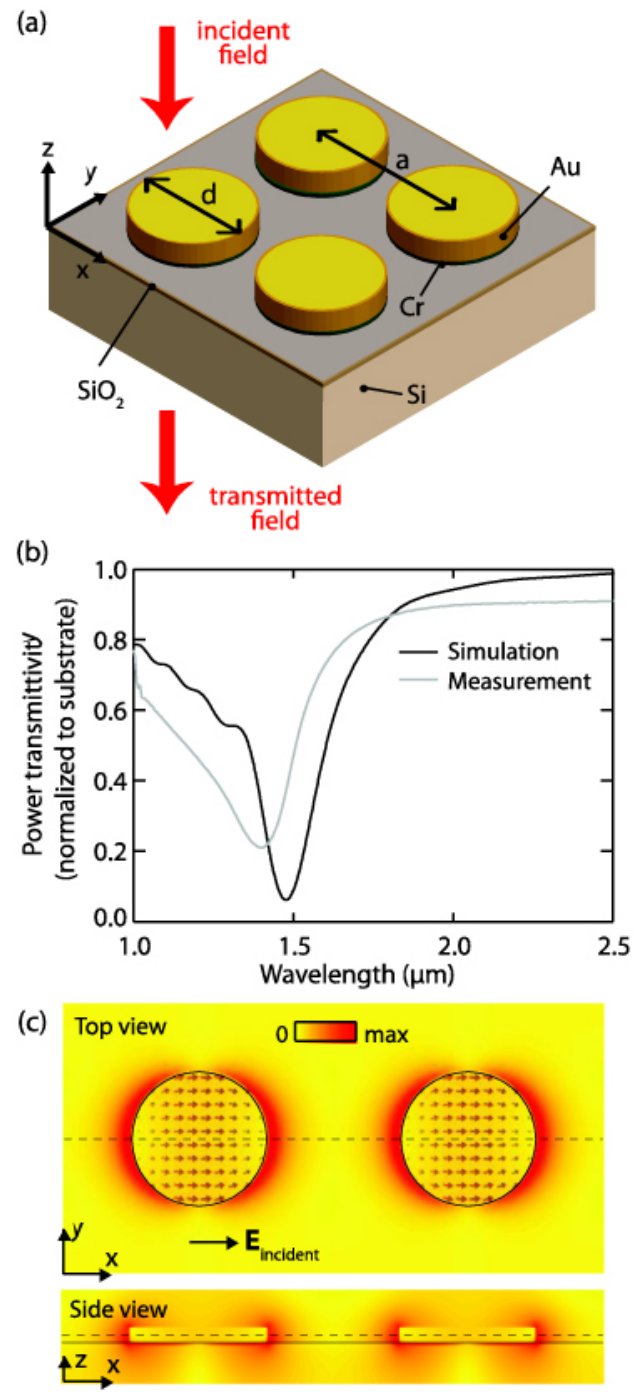

Fig. 1. (a) Geometric depiction of four gold nanodisks arranged in a square array on a silicon substrate. (b) Simulated and measured power transmission spectra normalized to the transmittivity of the substrate (c) Top and side views of the simulated electric field magnitude (color) and electric current density inside the metal (arrows) for the designed nanodisks at their resonance frequency, excited with normal incident light whose electric field is polarized along the $x$-direction. Side view uses a logarithmic scale in the field magnitude. The dashed line in each view indicates the cutplane shown in the other view.

Fabrication of the gold nanodisks was performed on a silicon substrate by standard electron-beam lithography with a single poly-methyl-methacrylate (PMMA). After developing of the resist, the sample was vacuum-deposited with a 1-2 nm chromium layer for adhesion purposed followed by a $20 \mathrm{~nm}$ gold layer. A subsequent lift-off process removed the residual resist. Figure 2 shows electron scanning microscope (SEM) images of the fabricated structure. The total sample area was $200 \times 200 \mu^{2}$. Figure 1(b) includes the transmission spectra of the sample, measured using a Bruker ${ }^{\mathrm{TM}}$ Fourier Transform IR spectrometer. The measured resonance frequency is slightly blue-shifted in comparison with the simulation. 


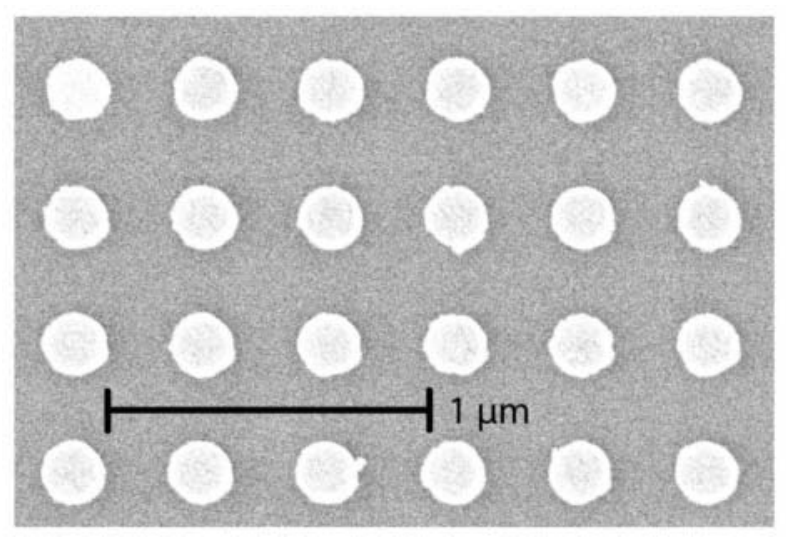

Fig. 2. Scanning electron microscope (SEM) image of the fabricated sample.

\section{Experimental measurements}

\subsection{Materials and methods}

Toluene 99.8\%, dichloromethane 99.5\%, 3-(triethoxysilyl) propyl isocyanate 3-(ICPTS) 95\%, isopropanol 99,5\%, ethylenediamine solution 75-80\% were obtained from Sigma -Aldrich. All solutions were made from particle free deionized (DI) water.

\subsection{Silicon surface functionalization}

Cleaning of the surface chip was carried out by rinsing with isopropanol and deionized (DI) water. Once the surfaces were cleaned and dried under a flow of dry air, 3-Isocyanatopropyltriethoxysilane (3-ICPTS) was used as self assembled monolayer (SAM) which serves as functionalization of the silicon surface for subsequent small molecule sensing. The procedure for the SAM formation was performed by immersion of the sample in a 1\% wt. solution of 3ICPTS in toluene for 2 hours to provide sufficient time for the self-assembled monolayer (SAM) to form. The deposition process was carried out at room temperature. In order to remove the excess reactants from both the silicon surface and the gold particles the sample was rinsed in dichloromethane and dried under a flow of dry air.

After the functionalization of the silicon surface, remove of the moisture from the active sites was performed by heating the sample first at $150{ }^{\circ} \mathrm{C}$ to regenerate the isocyanate group. Then, exposing the sample to ethylenediamine vapour at room temperature was carried out. Once the saturation level was reached, heating of the sensor at $150^{\circ} \mathrm{C}$ removed the amine molecules from the surface, yet left the isocyanate functional groups for subsequent amine binding events. Before the corresponding transmission measurement the sample was rinsed with DI water in order to remove the unbound molecules and dried under a flow of dry air.

\subsection{Infrared measurements}

Infrared (IR) transmission spectra for the characterization of the fabricated sample at each step were performed using a Bruker ${ }^{\text {TM }}$ Fourier Transform IR spectrometer. The IR illumination was focused on the sample with a $15 x$ objective with 0.4 numerical aperture, and the transmitted signal was collected with another microscope objective of same specifications on to a liquid nitrogen cooled mercury-cadmium telluride detector. The sample area of interest was spatially filtered with an adjustable square aperture in the microscope beam path to limit the transmitted signal to the $200 \times 200 \mu^{2}$ area of the sample. The illumination was unpolarised since the nanodisks LPSR is independent to the polarization of the incident radiation. When represented, each spectrum was normalized to the spectrum of the transmitted signal through a nearby part of the sample without nanodisks. 


\section{Results and discussion}

\subsection{Response of the LSPR to the SAM formation}

One of the key factors for success of a sensor is the construction of highly effective recognition surface capable of fast and reliable interaction with the corresponding binding analyte of interest. It is important to create a robust sensor surface with specific binding properties and minimal background interference to improve the screening capabilities. Cleaning processes of silicon substrates typically use piranha solution $\left(\mathrm{H}_{2} \mathrm{SO}_{4}: \mathrm{H}_{2} \mathrm{O}_{2}, 3: 1\right.$ by vol.) [20]. Anyway, we observed in our previous experiments that piranha solution damaged the gold nanodisks surfaces and changed slightly the LSPR peak of the gold nanostructure. For this reason, cleaning was carried out rinsing with isopropanol and DI water exclusively.

Figure 3 shows the transmission spectra before and after immersing the sample into the 3ICTPS solution for 2 hours. The spectrum shows that the minimum in the transmission blueshifted to $1.368 \mu \mathrm{m}$ in relation to the initial sample resonance of $1.396 \mu \mathrm{m}$. This observed notable blue-shift of $28 \mathrm{~nm}$ can be explained by a small increase in the nanoparticle height due to exposure to the solvent [21]. The formed monolayer is deposited in such a way that it covers completely the silicon surface, including the regions closely surrounding the gold particles. Organosilane SAM formation is driven by strong covalent attachment of these molecules to the native silicon oxide on the silicon surface through Si-O-Si bonds. The resulting 2-3 nm thick monolayer forms a densely packed and very stable structure with the isocyanate functional group oriented more or less along the normal to the silicon surface. Here, it is important to note that silanes do not form multilayers on gold surface at room temperature [22] and the rinse and heating steps should remove most of the deposited silane molecules from the gold surface, minimizing in this way the possible non-specific adsorption interference. Anyway, either SAM molecules or amine molecules could remain on the metal surface. To ensure that the non-specific adsorption of amine molecules was negligible, the bare sample prior to functionalization was exposed to amine vapour for a sufficiently long time and an IR measurement was carried out. Here, a LSPR wavelength red-shift of only $4 \mathrm{~nm}$ was obtained, which is negligible compared to the shifts obtained during sensing experiments. This means that there is no need to block the gold surface in order to avoid the non-specific adsorption. Therefore, sensing experiments were performed with no previous blocking of the gold surface, reducing thus cost in the fabrication process. It can be also pointed out that if a previous blocking of the gold surface was performed, the partially covered gold sidewalls could restrict sterically the subsequent SAM formation on the silicon areas close to the sidewalls, where the near-field is strongly confined, which would decrease dramatically the sensitivity of the sensor.

\subsection{Response of the LSPR to vapor exposure}

In order to evaluate the sensing ability of the silicon-functionalized system, we used ethylenediamine as target analyte, even though this molecule does not exhibit the greatest binding affinity constant to isocyanate groups. Sensing experiments were firstly performed by heating the sample at $150{ }^{\circ} \mathrm{C}$ on a hotplate to regenerate the isocyanate functional groups. This treatment removes the moisture from the surface, leaving the isocyanate functional groups available for the subsequent amine binding reaction. Then, the sample was exposed to ethylenediamine vapour for several minutes at room temperature. Before the transmission measurements, the sample was rinsed with deionised water in order to remove the unbound molecules and dried under a flow of dry air. As shown in Fig. 4a, binding of ethylenediamine molecules to isocyanate groups leads to significant red-shifts in the resonant wavelength of the nanodisks. Initially, the silicon surface modified with a monolayer of 3-ICTPS showed a transmission minimum at $1.368 \mu \mathrm{m}$ (black spectrum). After $5 \mathrm{~min}$ of exposure to ethylenediamine vapour we observed a wavelength red-shift to $1.392 \mu \mathrm{m}$. Increasing the exposure time to 15 and 25 min resulted in additional red-shifts to $1.403 \mu \mathrm{m}$ and $1.406 \mu \mathrm{m}$,

\#139457 - \$15.00 USD

(C) 2011 OSA 
respectively. After this time the resonance remained constant because the saturation level was reached. The sensor response evolves as a function of the analyte exposure time because the ethylenediamine binding at the functionalized surface is strongly time-depending. Hence, binding of ethylenediamine molecules at the isocyanate functional groups needs a determined incubation time to take place. The sensor response saturation occurs when the dynamic equilibrium is established between the sorption and desorption of molecules from the substrate. The binding kinetics will mainly depend on both the amount of active sites on the silicon surface and the number of analyte molecules in vapour phase. For this test, the time constant of the ethylenediamine vapour binding process, $\tau$, can be extracted from the characterization of the $\lambda_{\mathrm{LSPR}}$ shift, $\Delta \lambda_{\mathrm{LSPR}}$, as a function of the analyte exposure time, as shown in Fig. 4b. The experimental data were fitted by a time-dependent equation based on the sorption/desorption kinetics presented by Langmuir [23], $\Delta \lambda_{\mathrm{SPR}}=\Delta \lambda_{\max }[1-\exp (-\mathrm{t} / \tau)]$, where $\Delta \lambda_{\max }=37.8 \mathrm{~nm}$ was the sensor response corresponding to the saturation of the binding sites and the time constant $\tau=5.16 \pm 0.4 \mathrm{~min}$. This time depends upon the affinity reaction between the analyte and the isocyanate active sites on the silicon surface. Thus, the relative resonance shift $\Delta \lambda \lambda_{\max } / \lambda_{0}$, where $\lambda_{0}$ is the wavelength of the plasmonic resonance, can be determined in case of a complete saturation of the surface. A valu $\AA \lambda$ max $/ \lambda_{0}=0.027$ was obtained when all the actives sites were occupied by ethylenediamine molecules. Taking into account that only a small area of the silicon surface contributes strongly to the resonance shift, this approach provides a clear improvement in terms of sensitivity over other sensing techniques based on metal nanostructures [24].

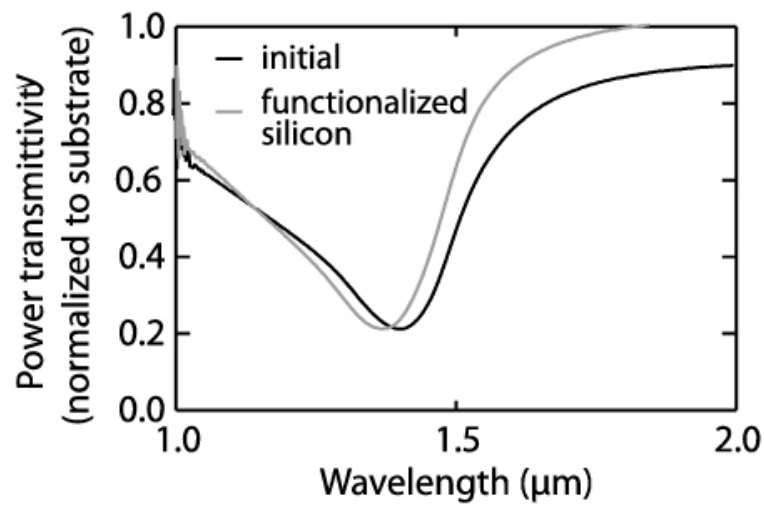

Fig. 3. Measured IR transmission spectra before and after chemical modification of the silicon surface.

\subsection{Surface regeneration}

One of the advantages of using isocyanate groups is that it allows an easy regeneration of the active sites, which is of key importance for the recycling of the same sensor platform [7]. Regeneration allows to reduce cost and to consume less time because of elimination of various preliminary steps like fabrication, calibration and standardization of the sensor surface. Moreover, multiple measurements with enhanced repeatability can be performed. Typically, surface regeneration is achieved by using chemical reactants such as HCl [25], $\mathrm{NaOH}[25,26]$, glycine-HCl [27] among others. They are in the $\mathrm{pH}$ range from highly acidic to basic and are used depending on the nature and extent of the binding. Anyway, these strong chemical reactants can affect the binding ability of the functionalized surface and could even damage the nanostructure by erosion of the metal, resulting in a general diminished lifetime of the chip. Our use of organosilane containing isocyanate as functional group has, however, the advantage of an easier and reliable regeneration of the active sites by a simple thermal step, avoiding the use of chemical reactants. Figure 5 shows how the heating of the sensor at $150^{\circ} \mathrm{C}$ regenerates the isocyanate, recovering approximately the signal corresponding to the functionalized sample. A subsequent sensing test was performed to check if the regenerated 
surface was still active, obtaining a smaller redshift of $14 \mathrm{~nm}$. This decrease in the resonance shift could possibly be caused by the damage to some actives sites during the regeneration and handling of the sample.
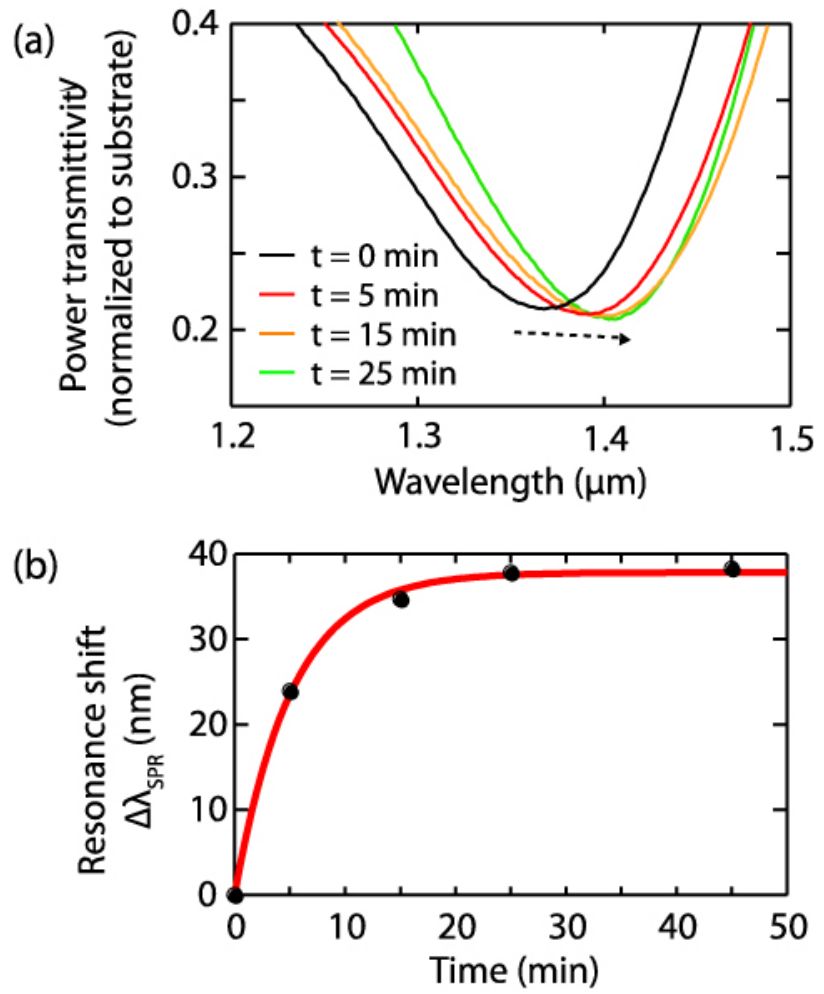

Fig. 4. (a) Transmission spectra at different exposure times to ethylenediamine vapour. (b) Saturation curve showing the resonance shift as a function of exposure time, and fitting to the Langmuir model. The spectrum at $45 \mathrm{~min}$ is not shown for the sake of clarity

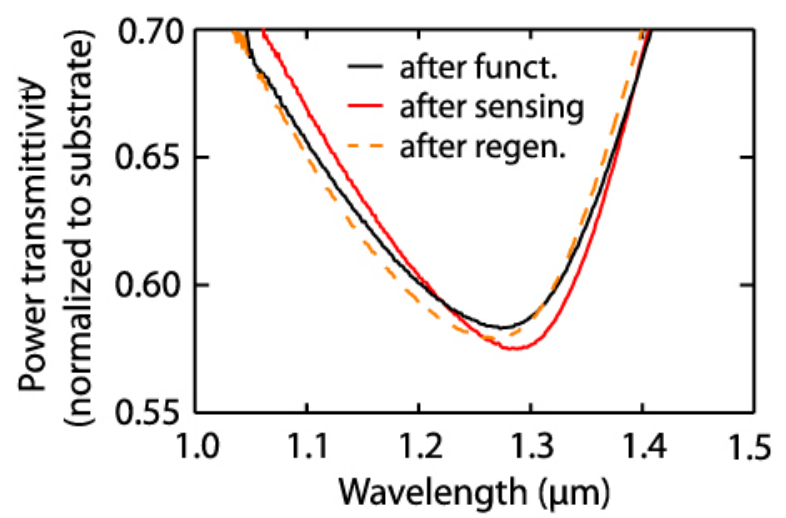

Fig. 5. Transmission spectra after the functionalization of the silicon surface (black curve), after sensing of ethylenediamine (red curve) and after heating at $150^{\circ} \mathrm{C}$ (dashed curve). Note that the power transmission levels differs from previous results due to the sample being from a different fabrication run.

\section{Conclusions}

In summary, we have demonstrated the use of LSPR of gold nanodisks on silicon in which we functionalized the silicon substrate surface for chemosensing purposes, unlike usual metal 
functionalization. This is possible thanks to the electric field of the nanodisk resonance being strong at the silicon surface surrounding it. The sensor was designed to show a strong resonance in the infrared regime, showing a high sensitivity of the system to SAM formation and subsequent binding events. The silicon surface instead of the gold surface was functionalized with 3-(ICPTS) containing isocyanate as functional group. The sensing ability of the system was investigated exposing it to amine vapour. Isocyanate-amine binding events led to significant red-shifts in the resonant wavelength up to $37 \mathrm{~nm}$ when the saturation level was reached. This value corresponds to a relative resonance shift $\Delta \lambda_{\max } / \lambda_{0}=0.027$. Taking advantages of the thermally reversible interaction isocyanate-amine, we demonstrated that the surface was easily regenerated by heating of the sensor, avoiding in this way the use of chemical reactants which could damage the metallic nanostructures. Further work will include the optimization of the metallic nanoparticle in order to maximize the SPR red-shift displacement when applying the organic substances.

\section{Acknowledgements}

Financial support by the Spanish MICINN under contracts CONSOLIDER EMET (CSD2008-00066) and TEC2008-06871-C02-02 and European Commission FP7 under the FET-Open project TAILPHOX 233833 is gratefully acknowledged. 